\title{
Characterizing Fentanyl-Associated Mortality using the Literal Causes of Death
}

\author{
Brandon Ramsey*, Heather Rubino, Janet J. Hamilton and David Atrubin \\ Florida Department of Health, Tallahassee, FL, USA
}

\section{Objective}

To characterize fentanyl-associated mortality in Florida using free text queries of the literal causes of death listed on death certificates.

\section{Introduction}

In October 2015, the Centers for Disease Control and Prevention (CDC) released health advisory \#384 to inform people about increases in fentanyl fatalities. Florida's statewide syndromic surveillance system, Electronic Surveillance System for the Early Notification of Community-based Epidemics (ESSENCE-FL), captures electronic death record data in near real time which allows for the monitoring of mortality trends across the state. One limitation of using death record data for fentanyl surveillance is the lack of a fentanyl-specific overdose ICD-10 code; however, the literal cause of death fields ("literals") provide a level of detail that is rich enough to capture mentions of fentanyl use. The "literals" are a free text field on the death certificate, recorded by a physician at the time of death and detail the factors that led to the death. ESSENCE-FL has the benefit of not only receiving death record data in near real-time, but also receiving the literal cause of death fields. This work analyzes trends in fentanyl-associated mortality in Florida over time by using the literal cause of death fields within death records data obtained from ESSENCE-FL.

\section{Methods}

The "literals" elements of Florida Vital Statistics mortality data from 2010 through 2015 accessed via ESSENCE-FL were queried for the term ${ }^{\wedge}$ fent ${ }^{\wedge}$. No necessary negations or extra term inclusions were deemed necessary after looking at the records pulled with ${ }^{\wedge}$ fent $^{\wedge}$ alone. Deaths were analyzed by various demographic and geographic variables to characterize this population in order to assess which groups are most heavily burdened by fentanyl-associated mortality. Population estimates by county for 2015 were obtained from the U.S. Census Bureau to calculate mortality rates. Language processing in $\mathrm{R}$ Studio was used to determine which other substances were commonly reported when fentanyl was listed on the death certificate, in order to assess polydrug use and its impact on increased mortality.

\section{Results}

Compared to the number of fentanyl-associated mortalities in 2010 (82), fentanyl-associated mortality in 2015 (599) was 6.5 times higher after controlling for the natural increase in total mortality between 2010 and 2015. Almost three-fourths of the deaths in 2015 were male $(73 \%)$, which is higher than the proportion of male deaths in 2010 (55\%). The age group with the largest burden of fentanyl-associated mortality was the $30-39$ age group, with almost one-third of the deaths in 2015 coming from this age group (31\%) compared to only $10 \%$ in 2010 , a roughly $200 \%$ increase. Fentanyl-associated mortality was almost exclusive to people that are Caucasian, with $94 \%$ of the fentanyl-associated mortalities in 2015 occurring among Caucasians. Multi-drug use was also identified for those with fentanyl-associated mortality. Mentions of other drugs were present in at least $10 \%$ of the deaths. Some of the other drugs mentioned in the "literals" included heroin, cocaine, and alprazolam. There was county variation in the number of fentanyl morality deaths ranging from 21.19 deaths per
100,000 to 0.29 deaths per 100,000 residents. Two counties with the highest rates were located adjacent to one another.

\section{Conclusions}

Having death record data readily available within the state syndromic surveillance system is beneficial for rapid analysis of mortality trends and the analytic methods used for syndromic surveillance can be applied to mortality data. Free text querying of the "literals" in the vital statistics death records data allowed for surveillance of fentanyl-associated mortality, similar to methods used for querying emergency department chief complaint data. Although underlying ICD-10 codes can lack detail about certain causes of death, the "literals" provide a clearer picture as to what caused the death. The "literals" also make it possible to look at potential drug combinations that may have increased risk of mortality, which will be explored more thoroughly. Further work will explore other data sources for fentanyl usage and mortality trends, as well as examine potential risk factors and confounders.

\section{Keywords}

Fentanyl; Mortality; Text Query

\section{*Brandon Ramsey}

E-mail: Brandon.Ramsey@flhealth.gov 